\title{
Protocolo Sanitario para Venados Cola Blanca (Odocoileus virginianus peruvianus) del Sector Sauce Grande - Coto de Caza El Angolo, Piura, Perú
}

\author{
Health Protocol for White Tailed Deer (Odocoileus Virginianus peruVianus) of \\ Sauce Grande Area of Coto de Caza El Angolo, Piura, Peru
}

Roberto Elías Piperis ${ }^{1,3}$, Pedro Vásquez Ruesta ${ }^{2}$

\section{Resumen}

\begin{abstract}
El venado cola blanca (Odocoileus virginianus peruvianus) es un rumiante de la familia Cervidae que se maneja como especie cinegética en el sector Sauce Grande, del Coto de Caza El Angolo (CCEA), Piura, Perú. El objetivo de este trabajo fue proponer un protocolo sanitario para venados cola blanca del CCEA como un componente esencial en sus planes de manejo y que pueda servir como modelo para otras especies silvestres en áreas naturales protegidas. Para el desarrollo de este protocolo se hizo una revisión bibliográfica sobre la especie y se realizaron cinco visitas al CCEA para el reconocimiento del área y la estandarización de algunos procedimientos, donde se tomaron muestras biológicas del venado cola blanca y de mamíferos domésticos de la zona para análisis microbiológicos.
\end{abstract}

Palabras clave: venado cola blanca, Odocoileus virginianus peruvianus, Coto de Caza El Angolo, protocolo sanitario

\section{AbstraCt}

The white-tailed deer (Odocoileus virginianus peruvianus) is a ruminant of the family Cervidae that is managed as a game species in the Sauce Grande area of Coto de Caza El Angolo (CCEA), Piura, Peru. The aim of this study was to propose a health protocol for the white-tailed deer of CCEA as an essential component of their management

\footnotetext{
${ }^{1}$ Laboratorio de Vida Silvestre, Facultad de Medicina Veterinaria y Zootecnia, Universidad Peruana Cayetano Heredia, Lima, Perú

${ }^{2}$ Centro de Datos para la Conservación, Facultad de Ciencias Forestales, Universidad Nacional AgrariaLa Molina,Lima,Perú.E-mail: cdc@lamolina.edu.pe

${ }^{3}$ E-mail: roberto.elias@upch.pe
}

Recibido: 18 de enero de 2016

Aceptado para publicación: 25 de abril de 2016 
work plans as well as to serve as a model for other wildlife in protected natural areas. For the development of this protocol a literature review was done on the species, along with five visits to CCEA for the evaluation of the area and the standardization of some procedures, where biological samples were collected from white-tailed deers and domestic mammals in the area for microbiologic analysis.

Key words: white-tailed deer, Odocoileus virginianus peruvianus, Coto de Caza El Angolo, health protocol

\section{INTRODUCCIÓN}

Una de las localidades donde se viene manejando fauna silvestre en el Perú es el Coto de Caza El Angolo (CCEA), específicamente en el sector Sauce Grande, donde se mantiene una población de venado cola blanca (Odocoileus virginianus peruvianus) bajo la administración del Club de Caza, Pesca y Turismo de Piura (CCPTP). El venado cola blanca es una de las nueve especies de cérvidos reportadas para el Perú y única del género Odocoileus en el país (Pacheco et al., 2009), con un amplio rango de distribución que va a lo largo del litoral, desde Piura hasta Tacna (Smith, 1991).

El CCEA fue establecido el 1 de julio de 1975 con la finalidad de ser utilizado para la conservación a través de la caza deportiva, aspecto reglamentado por el Ministerio de Agricultura (Brack et al., 1973). El CCEA es uno de los dos cotos de caza existentes en el Perú y es parte del Sistema Nacional de Áreas Naturales Protegidas por el Estado (SINANPE). Se encuentra en el departamento de Piura (distritos de Marcavelica y Lancones, provincia de Sullana, y en el distrito de Talara, provincia de Talara) y cuenta con 65000 ha, de las cuales 9918 ha corresponden al sector Sauce Grande (Vásquez y Justo, 2009).

En el 2005 se aprobó el primer plan maestro del CCEA y en 2007 el plan de manejo de venado de cola blanca para el periodo 2007-2010, siendo renovado en 2011 para el periodo 2011-2015 (Resolución de Jefatu- ra N. ${ }^{\circ}$ 001-2011-CCEA-SERNANP-JEF) (Vásquez y Justo, 2009).

El CCPTP, con ayuda de la Facultad de Ciencias Forestales y del Centro de Datos para la Conservación (CDC) de la Universidad Nacional Agraria La Molina (UNALM), ha realizado diversas evaluaciones sobre población, hábitat y dieta del venado (P. Vásquez, comunicación personal, Lima), pero aún no cuenta con programas de manejo sanitario.

El venado cola blanca, como miembro de la familia Cervidae, pertenece al suborden Ruminantia (orden Cetartiodactyla), grupo que comparte con bovinos, ovinos y caprinos, de allí que presentan muchas enfermedades en común, como brucelosis, leptospirosis y lengua azul, las cuales son de carácter zoonótico y, por lo tanto, pueden ser transmitidas a los seres humanos, constituyendo un problema de salud pública (Martínez et al., 1999). Por otro lado, el venado también puede actuar como hospedero definitivo para algunos agentes parasitarios, o como intermediario de parásitos de carnívoros silvestres, como Taenia hydatigena, T. omissa y Echinoccocus granulosus (Waid et al., 1985; Flach, 2003). No obstante, la información existente sobre agentes patógenos que afectan al venado cola blanca proviene mayormente de trabajos hechos en Norteamérica (Friend y Halterman, 1967; Davidson et al., 1985; Martínez et al., 1999; Miller et al., 2000).

El venado de cola blanca es el único recurso de la fauna silvestre que es aprovechado en el CCEA y tiene contacto con bovi- 
nos criollos, que son criados de manera extensiva por pobladores dentro y fuera del sector Sauce Grande, y con caprinos que se encuentran mayormente fuera del sector. La población de bovinos y caprinos en el CCEA es variable de año a año y no se lleva un control de la cantidad existente. Los bovinos se encuentran libres y únicamente son capturados para su beneficio, pues se han asilvestrado, dificultando su manejo y cualquier tipo de evaluación sanitaria que se pretenda realizar (R. Elías, datos no publicados).

Las enfermedades son unos de los factores ecológicos que se debe tener en cuenta en el manejo de una población de fauna silvestre (Wobeser, 2006) y deben ser consideradas en sus planes de manejo. Como ejemplo se puede mencionar el reporte técnico para el aprovechamiento de venado cola blanca, hecho por la institución Biocenosis para la Reserva Ría Lagartos, en Yucatán, México (Alcérreca, 1999), así como el plan de manejo para las zonas templadas y tropicales de México preparado por la Secretaría de Medio Ambiente, Recursos Naturales y Pesca (2007). En caso del plan maestro del CCEA no se incluye el manejo sanitario para esta especie.

Las poblaciones del venado cola blanca se han recuperado en el sector Sauce Grande a partir de 1992, luego de un periodo sin mayor control (1975-1992), con excepción de mortalidades registradas en 1976, pero sin reportar las causas.

El objetivo del presente trabajo fue proponer un protocolo sanitario para la población silvestre de venados cola blanca del sector Sauce Grande del CCEA que permita registrar de manera sistemática los eventos sanitarios y poder tomar medidas correctivas y preventivas. Esta propuesta pretende ser un aporte para la realización de evaluaciones zoosanitarias a futuro, teniendo en cuenta la importancia de este aspecto en el manejo de especies cinegéticas.

\section{Materiales y Métodos}

Se trabajó en el sector Sauce Grande, del Coto de Caza El Angolo, ubicado entre las provincias de Talara y Sullana, departamento de Piura, Perú, y la especie de la presente propuesta es el venado cola blanca, $O$. virginianus peruvianus (Gray 1874).

Se recopiló información sobre aspectos biológicos y reportes existentes sobre la salud de la especie, así como información sanitaria de rumiantes en el país, como base para formular la propuesta de protocolo sanitario.

Se revisaron los antecedentes ambientales (precipitación) del área con los datos obtenidos de la Estación Sauce Grande. Se revisaron las fotografías de cámaras trampa almacenadas en el CDC entre 2007 y 2013. Las cámaras trampa son colocadas por personal de la UNALM a lo largo de las trochas utilizadas para la práctica de cacería. Entre 2007 y 2012 solo fueron colocadas en los meses de noviembre y diciembre como parte del ejercicio de campo del Programa de Maestría de Conservación de Recursos Forestales y a partir del 2013 se mantienen todo el año. Entre 2010 y 2013 se registraron cerca de 50000 fotografías, aunque no todas fueron referentes al venado.

Se realizaron cinco viajes (julio, setiembre, diciembre 2011, y agosto, diciembre 2012). Se realizaron cuatro necropsias (una hembra juvenil, una hembra adulta, dos machos adultos) recolectándose 50 muestras biológicas (cerebro, corazón, pulmón, hígado, riñón, intestinos, órganos reproductores, músculo y hueso) en formol al $10 \%$ por cada cadáver para análisis histopatológico. Además, se recolectaron heces para análisis parasitológico y garrapatas de los cuatro venados, hisopados de tejidos infectados de dos ejemplares (hembra adulta y macho). Asimismo, heces de ganado bovino (1 muestra en 2011 y 83 en 2012) para análisis microbioló- 
gico (microorganismos aérobicos y anaérobicos) y frotices sanguíneos para descarte de hemoparásitos.

\section{Resultados y Discusión}

\section{Estado Situacional}

Las enfermedades de la fauna silvestre son un factor ecológico que muchas veces no se tienen en cuenta y que tienen una influencia directa sobre la dinámica poblacional de muchas especies. En este caso, se observaron cambios importantes en la población de venados cola blanca en 2011, estimándose la muerte de 300 individuos aproximadamente, pudiéndose verificar durante la realización de uno de los ejercicios de campo de los estudiantes del programa de maestría de la UNALM (P. Vásquez, comunicación personal, Lima). Este evento coincidió, además, con el inicio de las visitas del presente estudio.

A finales de 2010, a través de las fotografías de las cámaras trampa, se pudo determinar que varios ejemplares de venados cola blanca presentaban pelaje en mal estado, pudiéndose relacionar esta situación con una infestación masiva de garrapatas, observada durante el primer viaje en 2011.

Una fuerte sequía ocurrió en la región a inicios de 2011 y en junio se observó gran mortalidad de individuos juveniles, que fue en aumento en los meses siguientes, llegando a afectar también a ejemplares adultos ( $\mathrm{S}$. Saldarriaga, comunicación personal, Piura). Los animales presentaban cojera, diarrea y salivación previo a la muerte. En las cuatro necropsias realizadas se encontró sobrecrecimiento y asimetría de las pezuñas, con lesiones ulcerativas en la pared córnea y banda coronaria. En uno de los individuos, a nivel interdigital, se evidenció una herida profunda con presencia de material necrótico y abultamiento moderado de los metacarpos con secreción blanquecina purulenta, en tanto que las falanges distales presentaban desviación, confirmándose fractura completa y osteólisis mediante radiografía. El resultado de las muestras indicó bacilos Gram negativos fusiformes compatibles con Fusobacterium sp (Club de Caza, Pesca y Turismo - Piura, 2011).

En bovinos, Fusobacterium necrophorum es el principal agente etiológico causante de pododermatitis, infección necrotizante, aguda o crónica, de la piel interdigital y banda coronaria adyacente al tejido subcutáneo (Cardona y Cano, 2003; Togoe et al., 2008), aunque también se ha comprobado la existencia de un sinergismo con Actinomyces pyogenes (Nagaraja y Chengappa, 1998; Tadepalli et al., 2009) y asociación con Porphyromonas levii y Prevotella intermedia (Nagaraja et al., 2005).

Diversos reportes de mortalidad del venado de cola blanca mencionan como causa principal necrobacilosis y pododermatitis por F. necrophorum (Gavin et al., 1984; ChirinoTrejo et al., 2003; Haigh et al., 2005; Sieber et al., 2010). A. pyogenes ha sido reportada en un menor grado como causante de pododermatitis en esta especie (Nettles et al, 2002; Chirino-Trejo et al., 2003; Karns et al., 2009).

En este caso en particular se sospechó que el ganado bovino actuó como principal fuente del agente patógeno. El análisis microbiológico de una muestra de excremento de ganado dio positivo a F. necrophorum. Al parecer la sequía predispuso a la presentación de estos episodios, dada la escasez de recursos forrajeros, disminuyendo la condición corporal del ganado y de los venados. Esta condición pudo afectar el sistema inmunológico del ganado, causando una mayor presencia de la bacteria en las heces. La depresión del sistema inmune se pudo notar con la gran infestación de garrapatas Rhiphicephalus microplus que presentaban ambas especies. Asimismo, la falta de agua también hizo que ambas especies convergie- 
ran, compartiendo los pocos abrevaderos que se podían encontrar, lugares que presentaban abundante lodo mezclado con heces de vaca y agua empozada, por lo que los venados entraban en contacto directo con heces de vacas, según pudo comprobarse en las fotografías tomadas por las cámaras trampa (R. Elías, datos no publicados).

Otros factores que pudieron favorecer a la infección fue el desgaste y las heridas que comúnmente se hacen los venados con el suelo pedregoso del CCEA, aspecto que se observa en la mayoría de ejemplares cazados; además de heridas causadas por Tunga penetrans, que es un hallazgo frecuente en el ganado bovino y equino, y en venados cazados de la zona (P. Vásquez, comunicación personal, Lima), y que se puede acrecentar en animales débiles.

El CСТPP, a raíz de esta mortandad, adoptó algunas medidas de manejo que sirvieron para disminuir la exposición de los venados a la potencial fuente del patógeno. Se incineraron todos los cuerpos hallados, registrándose con GPS la ubicación de cada evento, y considerando el reducido número de aguadas, se crearon abrevaderos artificiales cercados para evitar el ingreso de vacas y se redujo el número de ganado (R. Elías, datos no publicados).

\section{Necesidad del Protocolo Sanitario}

La carencia de protocolo sanitario en áreas naturales hace que información valiosa se pierda y que, con el tiempo, no se puedan explicar algunos cambios en los ecosistemas. El mejor ejemplo fue lo ocurrido en el CCEA en 1976, que por conversaciones con los guías y ganaderos del lugar, se supo que hubo un gran episodio de los que ellos llaman «plaga» $\mathrm{o}$ «peste», que causó una gran mortalidad de venados, pero en la que no se recopiló ningún dato, tomándose únicamente la decisión de declarar una veda para caza de venados, veda que se mantendría a lo largo de los años sin evaluar sus efectos. Esta información perdida podría haberse uti- lizado para realizar una comparación con lo sucedido en el 2011 y saber el grado de relación de ambos eventos o para los cambios en la población de venados en esa época.

Según el Servicio Nacional de Sanidad Agraria (SENASA) del Perú, dentro de las enfermedades infecciosas reportadas para rumiantes domésticos, no se encuentran registros en venados cola blanca aunque existe la posibilidad de que podrían infectarlos. Algunas de esas enfermedades se encuentran en las Listas de la Organización Mundial de Sanidad Animal (OIE) y estas listas han sido declaradas por SENASA, bajo Resolución Jefatural N. ${ }^{\circ}$ 271-2008-AG-SENASA, como enfermedades notificables debido a su vital importancia en la vigilancia epidemiológica nacional. Algunas de estas enfermedades son consideradas zoonóticas (tuberculosis bovina, rabia, ántrax, etc.). La notificación de enfermedades de los animales es de carácter obligatorio y está establecido en el Artículo N. ${ }^{\circ} 9$ del Decreto Legislativo N. ${ }^{\circ} 1059$ «Ley General de Sanidad Agraria».

A partir de esta información obtenida del venado cola blanca en el CCEA se pudo notar que los factores que pueden considerarse de mayor riesgo para la aparición de enfermedades son la presencia de animales domésticos, el clima y los potenciales reservorios de enfermedades:

- Entre los animales domésticos, además del ganado bovino y caprino, se puede incluir a los perros de caza que pertenecen al CCPTP y algunos otros que son mascotas de los guías o que son usados para arrear al ganado. Además, en los alrededores del sector Sauce Grande se pudo observar cerdos, ovinos, aves de corral y gatos domésticos (R. Elías, observaciones no publicadas).

- La zona es bosque seco ecuatorial estacional y depende de las lluvias para el crecimiento de la vegetación y la acumulación de agua para los meses de seca. La estación húmeda tiene una duración de tres meses, coincidente con los me- 
ses de verano, y la seca es de nueve meses. En líneas generales, las lluvias están condicionadas por la corriente cálida o de El Niño, la presencia de la Cordillera de los Andes y su posición cercana a línea ecuatorial (Vásquez y Justo, 2009). Las precipitaciones registran desde $1537 \mathrm{~mm}$ anuales en el extremo NorOriental hasta valores de $47 \mathrm{~mm}$ en Talara o de $67 \mathrm{~mm}$ en Marcavelica (Vásquez y Justo, 2009).

- La presencia de potenciales reservorios silvestres de agentes infecciosos como, por ejemplo, el murciélago hematófago Desmodus rotundus, que es reservorio de rabia silvestre. Así mismo, de roedores como la ardilla nuca blanca (Sciurus stramineus), que podría servir de reservorio de leptospirosis (Montes et al., 2011).

\section{El Protocolo Sanitario}

El seguimiento y observación de los venados a lo largo de todas las trochas es relativamente fácil, a pesar de que estos se pueden «mimetizar» con el ambiente. No obstante, la observación debe hacerse manteniendo cierta distancia y con sigilo para evitar asustar a los ejemplares. Por eso y debido a la necesidad de incrementar la precisión de los métodos de monitoreo para evaluaciones poblacionales y estudios ecológicos (Voglioto et al., 2010), se recomienda incorporar técnicas de captura que también puedan ser útiles en evaluaciones sanitarias.

En este protocolo se recomienda el uso de cámaras trampa, que han demostrado efectividad como método de alerta temprana. Se pueden utilizar para inventarios de especies, estudios de uso de hábitat, comportamiento, patrones de actividad, recursos claves y dietas, aspectos reproductivos, parámetros demográficos (Voglioto et al., 2010) e, incluso, según lo observado en el CCEA, podría dar información sobre la condición corporal de los animales y de factores que pudieran estar influyendo en la aparición de ciertas enfermedades.
Existen técnicas directas que también se podrían utilizar como trampas, tales como las redes de cañón o jaulas trampa (Pooler et al., 1997; VerCauteren et al., 1997, 1999), aunque no existen antecedentes exitosos del uso de estos métodos en la zona de estudio. Un segundo método es la captura con ayuda de dispositivos para administración de fármacos a distancia; sin embargo, se deben evaluar los protocolos de contención química más adecuados, a fin de asegurar la captura de los animales considerando el tipo de topografía que tiene el CCEA en el sector Sauce Grande.

La necropsia es un procedimiento adecuado para determinar la causa de muerte de un animal, y debería realizarse en los venados cola blanca que se encuentren muertos. No obstante, es difícil hallar cadáveres en buen estado, ya que la mayoría, por acción del clima y de las especies carroñeras, tienden a deteriorarse con rapidez, de allí que se debería aprovechar la toma de muestras de los animales que son cazados (heces, sangre, ectoparásitos, tejidos y órganos internos) para análisis posteriores. Con la cuota de caza anual (100 ejemplares) se obtendría una muestra representativa de la población total del sector Sauce Grande (estimada en 1000 animales).

En resumen, el protocolo que se propone incluye principalmente: a) evaluaciones permanentes de los animales con la ayuda de las cámaras trampa, b) necropsias y toma de muestra de los cadáveres de venados encontrados en buen estado en el CCEA, c) evaluación y toma de muestras de animales cazados y d) registro de todo evento que incluya factor de riesgo como los mencionados en el texto.

\section{Conclusiones}

En el sector Sauce Grande, del Club de Caza, Pesca y Turismo de Piura (CCPTP), existen factores, tales como 
las variaciones en el clima, presencia de animales domésticos y de potenciales reservorios silvestres (murciélagos, ardillas) de agentes infecciosos, que constituyen un riesgo para la salud de los venados cola blanca (Odocoileus virginianus peruvianus).

- Las cámaras trampa han demostrado ser un instrumento valioso para registro de cambios en la condición corporal del venado cola blanca, sirviendo de sistema de alerta temprana.

- Se recomienda un protocolo sanitario para venados cola blanca mediante evaluaciones permanentes de los animales con el uso de cámaras trampa, necropsias, toma de muestras en animales cazados $\mathrm{y}$ registro de eventos de riesgo.

\section{Literatura Citada}

1. Alcérreca C. 1999. Aprovechamiento de venado cola blanca (Odocoileus virginianus) como estrategia para conservar áreas forestadas en la zona maya: Reserva Ría Lagartos, Yucatán. Biocenosis, México. [Internet]. Disponible en: http://www.conabio.gob.mx/ institucion/proyectos/resultados/ InfM029.pdf

2. Brack A, Ríos M, Reyes F. 1973. Evaluación y bases para el establecimiento de un coto de caza y un parque nacional en la cordillera de los Amotapes (PiuraTumbes). Lima: Ministerio de Agricultura - DGFF. $52 \mathrm{p}$.

3. Cardona J, Cano N. 2003. Alteraciones digitales en el ganado bovino del trópico bajo. MVZ Córdoba 8: 249-253.

4. Chirino-Trejo M, Woodbury MR, Huang F. 2003. Antibiotic sensitivity and biochemical characterization of Fusobacterium spp and Arcanobacterium pyogenes isolated from farmed white-tailed deer (Odocoileus virginia$n u s$ ) with necrobacillosis. J Zoo Wildl Med 34: 262-268. doi: 10.1638/02-019
5. Club de Caza, Pesca y Turismo de Piura. 2011. Informe anual 2011. Plan anual de manejo del Sector Sauce grande del Coto de Caza El Angolo (periodo enero-diciembre 2011). Piura: CCPTP. 15 p.

6. Davidson WR, Crum JM, Blue JL, Sharp DW, Phillips JH. 1985. Parasites, diseases, and health status of sympatric populations of fallow deer and white-tailed deer in Kentucky. J Wildl Dis 21: 153-159. doi: 10.7589/0090-355821.2.153

7. Flach E. 2003. Cervidae and Tragulidae. En: Fowler ME, Miller RE (eds). Zoo and wild animal medicine. $5^{\text {th }}$ ed. USA: Saunders. p 634-649.

8. Friend M, Halterman LG. 1967. Serologic surveys of two deer herds in New York States. Bull Wildlife Dis Assoc 3: $32-34$

9. Gavin TA, Suring LH, Vohs PA, Meslow EC. 1984. Population characteristics, spatial organization, and natural mortality in the Columbian WhiteTailed Deer. Wildl Monogr 91: 3-41.

10. Haigh J, Berezowski J, Woodbury MR. 2005. A cross-sectional study of the causes of morbidity and mortality in farmed white-tailed deer. Can Vet J 46: 507-512.

11. Karns GR, Lancia RA, Deperno CS, Conner MC, Stoskopf MK. 2009. Intracranial abscessation as a natural mortality factor for adult male whitetailed deer (Odocoileus virginianus) in Kent County, Maryland, USA. J. Wildl Dis 45: 196-200.

12. Martínez A, Salinas A, Martínez F, Cantu A, Miller DK. 1999. Serosurvey of selected disease agents in white tailed deer of Mexico. J Wildl Dis 35: 799-803. doi: 10.7589/0090-3558-35.4.799

13. Miller MW, Williams ES, McCarty CW, Spraker TR, Kreeger TJ, Larsen CT, Thorne T. 2000. Epizootiology of chronic wasting disease in free-ranging in cervids in Colorado and Wyoming. J Wildl Dis 36: 676-690. 
14. Montes D, Rivera H, Ramírez M, Ríos P, Angulo C, Muñoz K. 2011. Frecuencia de infección por Leptospira sp en ardillas nuca blanca (Sciurus stramineus) en un zoológico de la ciudad de Lima. Rev Inv Vet Perú 22: 6771. doi: 10.15381/rivep.v22i1.124

15. Nagaraja TG, Chengappa MM. 1998. Liver abscesses in feedlot cattle: a review. J Anim Sci 76: 287-298.

16. Nagaraja TG, Narayanan SK, Stewart GC, Chengappa MM. 2005. Fusobacterium necrophorum infections in animals: pathogenesis and pathogenic mechanisms. Anaerobe 11: 239-246. doi: 10.1016/j.anaerobe.2005.01.007

17. Nettles VF, Quist CF, Lopez RR, Wilmers TJ, Frank P, Roberts W, et al.. 2002. Morbidity and mortality factors in key deer (Odocoileus virginianus clavium). J Wildl Dis 38: 685-692.

18. Pacheco V, Cadenillas R, Salas E, Tello C, Zeballos H. 2009. Diversidad y endemismo de los mamíferos del Perú. Rev Peru Biol 16: 5-32. doi: 10.15381/ rpb.v16il.111

19. Pooler RL, Curtis PD, Richmond MD. 1997. Cost comparisons for white-tailed deer live capture techniques. In: Proc VIII Eastern Wildlife Damage Management Conference. Roanoke, Virginia.

20. Sieber V, Robert N, Schybli M, Sager $H$, Miserez R, Engels M, RyserDegiorgis. MP. 2010. Causes of mortality and diseases in farmed deer in Switzerland. Vet Med Int 8: 781-789. doi: 10.4061/2010/684924

21. Smith WP. 1991. Odocoileus virginianus. Mamm Species 388: 1-13.

22. Tadepalli S, Narayanan SK, Stewart GC, Chengappa MM, Nagaraja TG. 2009. Fusobacterium necrophorum: a ruminal bacterium that invades liver to cause abscesses in cattle. Anaerobe 15: 36-43. doi: 10.1016/j.anaerobe. 2008. 05.005

23. Togoe I, Tudor L, Togoe D, Tudor L, Galis $A$. 2008. Investigations concerning the isolation of Fusobacterium necrophorum stems from podal affections of cattle. Lucr Stiint Med Vet 41:960-966.

24. Vásquez P, Justo M. 2009. La fauna silvestre del Coto de Caza El Angolo. Guía para la identificación de las aves. Lima: Centro de Datos para la Conservación - Universidad Nacional Agraria La Molina. 201 p.

25. VerCauteren $K C$, Beringer $J$, Hygnstrom SE. 1997. Use of netted cage traps in population management and research of urban white-tailed deer. [Internet]. Available in: http://digitalcommons.unl.edu/cgi/viewcontent. cgi?article $=1379 \&$ context $=$ gpwdcwp

26. VerCauteren $K C$, Beringer $J$, Hygnstrom SE. 1999. Use of netted cage traps for capturing white-tailed deer. [Internet]. Available in: http:// digit a $1 \mathrm{commons}$. un 1 . edu/ icwdm_usdanwrc/833/

27. Voglioto A, Varela DM, Andriolo A. 2010. Camera traps. In: Barbanti JM, Gonzalez S (eds). Neotropical cervidology, biology and medicine of Latin American deer. Jaboticabal, SP, Brasil: FUNEP- IUCN. p 296-305.

28. Waid DD, Pence DB, Warren RJ. 1985. Effects of season and physical condition on the gastrointestinal helminth community of white-tailed deer from the Texas Edwards Plateu. J Wildl Dis 21: 264-273.

29. Wobeser GA. 2006. Fundamentos de las enfermedades de los animales silvestres. España; Acribia. 284 p. 